\title{
Output Feedback Stabilization of Discrete Time Networked Systems with External Disturbance
}

\author{
Eloy Garcia and Panos J. Antsaklis
}

\begin{abstract}
We consider the problem of stabilization and disturbance rejection in discrete time systems with limited feedback information as it is commonly the case in Networked Control Systems. In a networked system, transmission of information is constrained by the network bandwidth and availability. Our goal is to decrease the rate at which a perturbed system needs to transmit feedback information to update its controller node while maintaining stability and disturbance rejection properties. A state-disturbance observation approach is presented and we show in this paper that asymptotic stability can be obtained in the presence of constant disturbances. Additionally, we provide conditions for inter-update performance for both, constant and piece-wise constant disturbances.
\end{abstract}

\section{INTRODUCTION}

$\mathbf{I}_{\mathrm{c}}^{\mathrm{N}}$ Networked Control Systems (NCS) a digital communication network is used to transfer information among the components of a control system including actuators, controllers, and sensors. NCS differ significantly from classical control systems where all system components are attached directly to the control plant exchanging information using dedicated wiring [1]. The use of a shared communication channel for control systems makes feedback measurements inaccessible to the controller for long intervals of time. An approach followed by different authors is to keep the latest received feedback measurement constant until new information arrives from the controller, i.e. using a Zero-Order-Hold (ZOH), [2]-[4].

The use of a model of the system in the controller node provides better performance in general since an estimate of the state of the system can be used for control during the time intervals that feedback measurements are not available.

Model-based frameworks have been used by different authors for control of networked systems [5]-[10], [12]-[17] or for control of systems with limited feedback communication [11]. The work in [5]-[11] considers model uncertainties and no external disturbances while the authors of [12]-[13] focus on attenuation of constant input disturbances by considering no plant-model mismatch. Similar model-based frameworks have been studied by different authors, for instance, the work in [14] considers discrete time systems subject to packet dropouts and the model dynamics are used to generate the control inputs when no feedback measurements are successfully received.

Eloy Garcia is a contractor (Infoscitex Corp.) with the Control Science Center of Excellence, Air Force Research Laboratory, WPAFB, OH 45433, USA. Corresponding author: elgarcia@infoscitex.com.

Panos J. Antsaklis is with the Department of Electrical Engineering, University of Notre Dame, Notre Dame, IN 46556 USA.

Partial support of the National Science Foundation under Grant No. CNS-1035655 is gratefully acknowledged.
The authors of [15] and [16] used the model-based framework in [5] for, respectively, stabilization of singularly perturbed systems and stabilization of coupled networked uncertain systems.

In the present paper we use the state/disturbance observation approach presented in [18]. The work in [18] discussed observability and stability conditions for continuous time systems but inter-update performance was not addressed. This is an important issue since inter-update performance is degraded, in general, using long update periods. In the present paper we consider discrete-time systems and, more importantly, we present a design procedure to select the update time period in order to obtain a desired performance for the inter-update response of the system. The results in this paper address the case of disturbance attenuation and consider the situation of output feedback using state observers. We implement an augmented state observer that provides estimates not only of the states of the disturbed system but also estimates of the unknown external disturbance. We provide observability conditions for the augmented system in terms of the original plant parameters. Then, this augmented state observer is used in the networked system and we show that asymptotic stability can be achieved for any finite update period in the presence of constant disturbances. This stability result is made possible by implementing a model-based framework that includes an estimate of the constant disturbance to compute the control input and by including an augmented observer system that asymptotically converges to the real state and disturbance.

Since the inter-update response may be poor when using very long update periods we focus our attention to system performance between update intervals and derive conditions on the choice of the update interval in order to establish bounds on the response of the system. Here, we consider both, constant and piece-wise constant disturbances. The use of the combined model-based approach and the augmented observer considerable improves the performance of the networked system compared to similar work in [12]-[13].

The paper is organized as follows. Section II introduces the model-based architecture for control over networks. Section III describes the augmented state observer for discrete time systems and provides observability conditions on the augmented system. The main results of the paper are given in Section IV. First, we show that asymptotic stability in the presence of constant disturbances can be obtained using any finite update interval. Then, we analyze the interupdate performance of the system for both constant and piece-wise constant disturbances. Examples are presented in Section V and Section VI concludes the paper. 


\section{PROBLEM DESCRIPTION}

The particular networked configuration that we implement in this paper is the Model-Based Networked Control System (MB-NCS) architecture that was presented in [5]. The MBNCS configuration makes use of an explicit model of the plant which is added to the actuator/controller node to compute the control input based on the state of the model rather than on the plant state. The state of the model is updated when the controller receives the measured state of the plant that is sent from the sensor node every $h$ seconds, where $h=k_{\mu+1}-k_{\mu}$ is constant (and also an integer for the case of discrete-time systems) and $k_{\mu}$ represents the time instants when a measurement is transmitted from the sensor node to the controller node. This notation emphasizes the fact the transmissions do not occur at every time $k=0,1,2 \ldots$ but at some of those time instants specified by $k_{\mu}$.

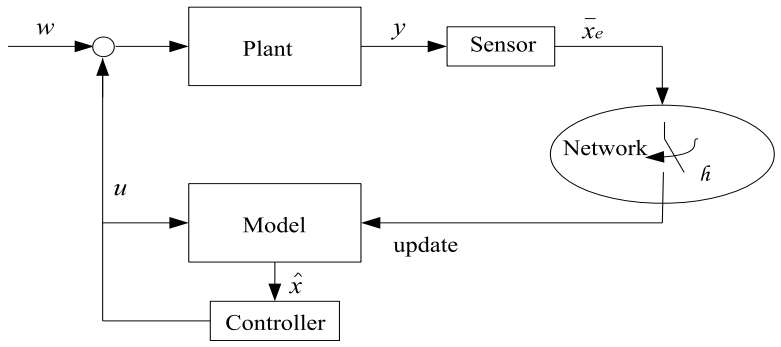

Fig. 1. Model-based networked system with external disturbance

Consider a Model-Based Networked Control System (MB-NCS) as shown in Fig. 1. An important difference with respect to the MB-NCS setup used in [5]-[10] is the consideration of an external disturbance $w(k)$. Assume that the disturbance is a persistent disturbance; in particular, we assume that it is a constant signal. This approach can be used in practice for piece-wise constant disturbances. In this case the output of the system is not asymptotically stable since there could be infinite number of discrete changes on the disturbance as time goes to infinity. However, bounded stability can be obtained as it is shown in this paper.

\section{AUGMENTED STATE OBSERVER}

Consider a dynamical system represented by discrete time equations of the following form:

$$
\begin{aligned}
& x(k+1)=A x(k)+B(u(k)+w(k)) \\
& y(k)=C x(k)
\end{aligned}
$$

where $x(k) \in \mathbb{R}^{n}, u(k) \in \mathbb{R}^{m}$, and $y(k) \in \mathbb{R}^{p}$, represent the state, control input, and measurable output of the system, respectively. $w(k) \in \mathbb{R}^{m},\|w(k)\| \leq W$ represents the unknown constant external disturbance. In this section, we focus on the state and disturbance estimation of (1). In Section IV this augmented state observer will be implemented in the sensor block shown in Fig. 1. We assume, for the moment, that both, the control input $u(k)$ and the system output $y(k)$ are available at the augmented observer for all times $k=0,1,2, \ldots$
Particular implementation details corresponding to the networked configuration as shown in Fig. 1 will be discussed in Section IV. Define the augmented state vector:

$$
x_{e}(k)=\left[\begin{array}{ll}
x(k)^{T} & w(k)^{T}
\end{array}\right]^{T} .
$$

The augmented system is given by:

$$
\begin{aligned}
x_{e}(k+1) & =A_{e} x_{e}(k)+B_{e} u(k) \\
y(k) & =C_{e} x_{e}(k)
\end{aligned}
$$

where

$$
A_{e}=\left[\begin{array}{cc}
A & B \\
0 & I
\end{array}\right], B_{e}=\left[\begin{array}{l}
B \\
0
\end{array}\right], C_{e}=\left[\begin{array}{ll}
C & 0
\end{array}\right] .
$$

The state observer for the augmented system (3) is given by:

$$
\bar{x}_{e}(k+1)=\left(A_{e}-L C_{e}\right) \bar{x}_{e}(k)+B_{e} u(k)+L y(k) .
$$

where $L$ is a stabilizing observer gain for the augmented system, that is, the eigenvalues of $A_{e}-L C_{e}$ satisfy $\left|\lambda_{L i}\left(A_{e}-L C_{e}\right)\right|<1$ for $i=1, \ldots, n+m$.

Theorem 1. Assume the discrete time pair $(A, C)$ is observable, then $\left(A_{e}, C_{e}\right)$ is observable if $\mathcal{N}(M)=\{0\}$, the nullspace of $M$ contains only the zero vector, where

$$
M=\left[\begin{array}{cc}
A-I & B \\
C & 0
\end{array}\right] .
$$

Proof. Let $\lambda_{i}^{e}$ represent the eigenvalues of $A_{e}$ for $i=1, \ldots, n+m$, with associated eigenvectors $v_{i}^{e}$. Note that

$$
\begin{array}{ll}
\lambda_{i}^{e}=\lambda_{i} & \text { for } i=1, \ldots, n . \\
\lambda_{i}^{e}=1 & \text { for } i=n+1, \ldots, n+m .
\end{array}
$$

where $\lambda_{i}$ represents the eigenvalues of $A$ for $i=1, \ldots, n$, and the associated eigenvectors to $\lambda_{i}$ are given by $v_{i}$. For any $\lambda_{i}^{e} \neq 1$ we have that:

$$
\left(\lambda_{i}^{e} I-A_{e}\right) v_{i}^{e}=\left[\begin{array}{cc}
\lambda_{i} I-A & -B \\
0 & \left(\lambda_{i}-1\right) I
\end{array}\right]\left[\begin{array}{l}
v_{n} \\
v_{m}
\end{array}\right]=0
$$

since $\lambda_{i} \neq 1$ then we have that

$$
\left(\lambda_{i}-1\right) v_{m}=0
$$

results in $v_{m}=0$ and

$$
\left(\lambda_{i} I-A\right) v_{n}=0 \Rightarrow v_{n}=v_{i} \Rightarrow v_{i}^{e}=\left[\begin{array}{l}
v_{i} \\
0
\end{array}\right] .
$$

Additionally, assume that $\lambda_{i}^{e} \neq 1$ is an unobservable eigenvalue of $\left(A_{e}, C_{e}\right)$, then by the $\mathrm{PBH}$ unobservable eigenvalue condition [19] we have that:

$$
\left[\begin{array}{c}
\lambda_{i}^{e} I-A_{e} \\
C_{e}
\end{array}\right] \hat{v}=0
$$

for some $\hat{v} \neq 0$. Equation (11) can be expressed as:

$$
\left[\begin{array}{cc}
\lambda_{i} I-A & -B \\
0 & \left(\lambda_{i}-1\right) I \\
C & 0
\end{array}\right]\left[\begin{array}{l}
\hat{v}_{n} \\
\hat{v}_{m}
\end{array}\right]=0
$$

which results in $\hat{v}_{m}=0$ and

$$
\left[\begin{array}{c}
\left(\lambda_{i} I-A\right) \hat{v}_{n} \\
C \hat{v}_{n}
\end{array}\right]=0
$$


but since $(A, C)$ is observable (13) is only satisfied by $\hat{v}_{n}=0$ then we have a contradiction and consequently any $\lambda_{i}^{e} \neq 1$ is an observable eigenvalue of $\left(A_{e}, C_{e}\right)$.

Now consider $\lambda_{i}^{e}=1$ and, similarly, assume that $\lambda_{i}^{e}=1$ is an unobservable eigenvalue of $\left(A_{e}, C_{e}\right)$, then we have that (11) is satisfied for some $\hat{v} \neq 0$. Equation (11) now results in the following:

$$
\left[\begin{array}{c}
(I-A) \hat{v}_{n}-B \hat{v}_{m} \\
C \hat{v}_{n}
\end{array}\right]=0
$$

which can also be expressed as

$$
M\left[\begin{array}{l}
\hat{v}_{n} \\
\hat{v}_{m}
\end{array}\right]=0
$$

but from the condition on $M$ expressed in the theorem, we have that (15) can only be satisfied by $\hat{v}=\left[\begin{array}{ll}\hat{v}_{n}^{T} & \hat{v}_{m}^{T}\end{array}\right]^{T}=0$ and we have a contradiction, therefore any $\lambda_{i}^{e}=1$ is an observable eigenvalue of $\left(A_{e}, C_{e}\right)$ and the augmented system (3) is observable.

\section{MODEL-BASED CONTROL WITH LIMITED COMMUNICATION}

The networked system in Fig. 1 contains a nominal model of the system in order to generate an estimate of the state of the disturbed system (1) and to compute the control input between update intervals. The model dynamics are given by:

$$
\begin{aligned}
\hat{x}(k+1) & =A \hat{x}(k)+B \hat{u}(k) \\
\hat{y}(k) & =C \hat{x}(k) .
\end{aligned}
$$

where $\hat{u}(k)=K \hat{x}(k)$. The use of the augmented observer described in the previous section provides an estimate of the unknown external disturbance that can be transmitted to the controller node in order to improve the control action by compensating for the effects of the disturbance $w(k)$, that is, the control input for the plant is given by:

$$
u(k)=K \hat{x}(k)-\hat{w}(k)
$$

where $K$ is a stabilizing gain for the nominal system (16), that is, the eigenvalues of $A+B K$ satisfy $\left|\lambda_{K i}(A+B K)\right|<1$ for $i=1, \ldots, n$, and $\hat{w}(k)$ represents the latest update of the disturbance estimate received at the controller node. i.e.

$$
\hat{w}(k)=\bar{w}\left(k_{\mu}\right) \quad \text { for } k \in\left[k_{\mu}, k_{\mu+1}\right)
$$

where $k_{\mu}$ represents the update instants and $k_{\mu+1}-k_{\mu}=h \geq 1$ is the update period and it is assumed to be constant, i.e. we use periodic communication. The update period $h$ represents the number of time units, as measured by the discrete time index $k$ of the underlying system (1), that the sensor waits in order to transmit a new observer state since the last update took place. Since the discrete-time index is the set of nonnegative integers, then it is clear that $h$ is an integer. At the update instants the augmented observer state $\bar{x}_{e}\left(k_{\mu}\right)$ is received at the controller node. The observer state contains estimates of both, the states of the plant and the disturbance. The observed disturbance is kept constant in the controller node during the next update interval as described in (18) and the model state is updated using the part of the observer state corresponding to the estimated plant states, i.e.

$$
\hat{x}\left(k_{\mu}\right)=\bar{x}\left(k_{\mu}\right) \text {. }
$$

After a model update takes place the model keeps its normal execution as in (16).

The sensor node contains the augmented observer and it has access to the output of the system at all times $k=0,1,2, \ldots$. In order to provide the observer with the control input $u(k)$ a copy of the model is implemented in the sensor node which is updated using the observer state at the same time instants that the model in the controller. Define the state error:

$$
\hat{e}(k)=x_{e}(k)-\left[\hat{x}(k)^{T} \hat{w}(k)^{T}\right]^{T} .
$$

Define the state vector $z(k)$ that contains the state of system (1), the state error defined in (20), and the observer state error, that is,

$$
z(k)=\left[\begin{array}{lll}
x(k)^{T} & \hat{e}(k)^{T} & e(k)^{T}
\end{array}\right]^{T}
$$

where

$$
e(k)=x_{e}(k)-\bar{x}_{e}(k) .
$$

The evolution of (21) during the time interval $k \in\left[k_{\mu}, k_{\mu+1}\right)$ is given by:

where

$$
z(k+1)=\Lambda z(k)
$$

$$
\Lambda=\left[\begin{array}{crc}
A+B K & {[-B K} & B] \\
0 & A_{e} & 0 \\
0 & 0 & A_{e}-L C_{e}
\end{array}\right]
$$

At the update instants we have that the model variables $\left[\hat{x}(k)^{T} \hat{w}(k)^{T}\right]^{T}$ are updated using the observer state $\bar{x}_{e}(k)$, that is, the following updates take place:

$$
\begin{aligned}
& x\left(k_{\mu}\right)=x\left(k_{\mu}^{-}\right) \\
& \hat{e}\left(k_{\mu}\right)=e\left(k_{\mu}^{-}\right) \\
& e\left(k_{\mu}\right)=e\left(k_{\mu}^{-}\right) .
\end{aligned}
$$

Theorem 2. The system described by (23) with periodic updates (25) is asymptotically stable for any constant disturbance $\|w(k)\| \leq W$ and for any finite update time $h \geq 1$ if $(A, B)$ is stabilizable, $(A, C)$ is observable and the nullspace of (6) contains only the zero vector.

Proof. Without loss of generality consider $k_{0}=0$. On the interval $k \in\left[0, k_{1}\right)$ the response of the system is given by:

$$
z(k)=\Lambda^{k} z_{0} \text {. }
$$

At $k=k_{1}$ the state of the model is updated using the state of the observer, this can be represented as follows:

$$
z\left(k_{1}\right)=\left[\begin{array}{ccc}
I & 0 & 0 \\
0 & 0 & I \\
0 & 0 & I
\end{array}\right] \Lambda^{h} z_{0} .
$$

Continuing with the interval $k \in\left[k_{1}, k_{2}\right)$ we have:

$$
z(k)=\Lambda^{k-k_{1}}\left[\begin{array}{ccc}
I & 0 & 0 \\
0 & 0 & I \\
0 & 0 & I
\end{array}\right] \Lambda^{h} z_{0}
$$


At $k=k_{2}$ the state of the model is updated once again using the state of the observer, and we have the following:

$$
z\left(k_{2}\right)=\left(\left[\begin{array}{ccc}
I & 0 & 0 \\
0 & 0 & I \\
0 & 0 & I
\end{array}\right] \Lambda^{h}\right)^{2} z_{0}
$$

By following the same analysis at every cycle $k \in\left[k_{\mu}, k_{\mu+1}\right)$ we obtain that the response of the system given by

$$
z(k)=\Lambda^{\left(k-k_{\mu}\right)}\left(\left[\begin{array}{ccc}
I & 0 & 0 \\
0 & 0 & I \\
0 & 0 & I
\end{array}\right] \Lambda^{h}\right)^{\mu} z_{0} .
$$

The term $\Lambda^{\left(k-k_{\mu}\right)}$ has a finite growth since $k-k_{\mu}<h$, then we analyze the properties of the matrix inside the brackets in (30) to determine the stability of the system. Note that this matrix is of the form

$$
\left[\begin{array}{lll}
I & 0 & 0 \\
0 & 0 & I \\
0 & 0 & I
\end{array}\right] \Lambda^{h}=\left[\begin{array}{ccc}
(A+B K)^{h} & Y & 0 \\
0 & 0 & \left(A_{e}-L C_{e}\right)^{h} \\
0 & 0 & \left(A_{e}-L C_{e}\right)^{h}
\end{array}\right]
$$

The eigenvalues of (31) are given by the eigenvalues of $(A+B K)^{h}$ (which are given by $\left.\left(\lambda_{K i}\right)^{h}\right), n+m$ eigenvalues at zero, and the eigenvalues of $\left(A_{e}-L C_{e}\right)^{h}$ (which are given by $\left.\left(\lambda_{L i}\right)^{h}\right)$. Since both sets of eigenvalues $\lambda_{K i}, \lambda_{L i}$, have magnitude less than one, then all eigenvalues of (31) have magnitude less than one and the system is asymptotically stable.

\section{A. Inter-sample performance analysis.}

The result in Theorem 2 shows that it is possible to asymptotically stabilize an open-loop unstable system with an unknown constant disturbance by using the model-based approach described in this section and reducing the frequency of feedback updates. However, the performance between updates could significantly deteriorate for very long update intervals. In the following we determine bounds on the performance of the system as a function of the update time intervals.

Consider the response of the system in the following form.

$$
x(k+1)=(A+B K) x(k)+[-B K B] \hat{e}(k)
$$

At the update time instants $k_{\mu}$ we have that $\hat{e}\left(k_{\mu}\right)=e\left(k_{\mu}^{-}\right)$, then we can write

$$
x(k+1)=(A+B K) x(k)+[-B K B] A_{e}^{k-k_{\mu}} e\left(k_{\mu}\right)
$$

The response of (33) can be expressed as

$$
x(k)=(A+B K)^{k-k_{\mu}} x\left(k_{\mu}\right)+\Phi e\left(k_{\mu}\right)
$$

for $k \in\left[k_{\mu}, k_{\mu+1}\right)$, where

$$
\Phi=\sum_{i=0}^{k-k_{\mu}-1}(A+B K)^{k-k_{\mu}-1-i}\left[\begin{array}{ll}
-B K & B
\end{array}\right] A_{e}^{i}
$$

In order to obtain a desired performance using this approach it is necessary to allow sufficient time for the observer to converge, within some specified bounds, and then transmit its state to update the controller. This means that we will avoid the use of relatively short update time intervals. This situation is in fact in line with the main purpose of the paper which is to reduce communication between the sensor node and the controller node. A similar condition will be considered to allow the system to operate with a new set of updated state and disturbance estimates for a sufficient time before the model variables are updated again.

Corollary 3. Assume that $\|x(0)\| \leq \gamma$ and the observer and model states are initialized as $\bar{x}_{e}(0)=0, \hat{x}(0)=0, \hat{w}(0)=0$. Then, the response of the system between updates is bounded by the following:

$$
\|x(k)\| \leq \varepsilon_{K} \gamma+\|\Phi\|(\gamma+W)
$$

if $(A, B)$ is stabilizable, $(A, C)$ is observable and the nullspace of (6) contains only the zero vector, where $\varepsilon_{K}+\varepsilon_{L}<1$ and the update time interval $h$ satisfy $\left\|(A+B K)^{h}\right\| \leq \varepsilon_{K}$ and $\left\|\left(A_{e}-L C_{e}\right)^{h}\right\| \leq \varepsilon_{L}$, for some $\varepsilon_{K}, \varepsilon_{L}>0$.

Proof. Since all eigenvalues of matrix $A_{e}-L C_{e}$ have magnitude less than one, then there exists some update interval $h\left(\varepsilon_{L}\right)$ such that $\left\|\left(A_{e}-L C_{e}\right)^{h}\right\| \leq \varepsilon_{L}$ for any $h \geq h\left(\varepsilon_{L}\right)$. Similarly, there exists some update interval $h\left(\varepsilon_{K}\right)$ such that $\left\|(A+B K)^{h}\right\| \leq \varepsilon_{K}$ for any $h \geq h\left(\varepsilon_{K}\right)$. Then, for some choice of $\varepsilon_{K}, \varepsilon_{L}$, such that $\varepsilon_{K}+\varepsilon_{L}<1$, we define $h=\max \left(h\left(\varepsilon_{K}\right), h\left(\varepsilon_{L}\right)\right)$. We can analyze how much the norm of the state could grow between update intervals by studying the growth of the state of the system at times $k_{\mu}^{-}$, before the update times, as follows:

$$
\begin{aligned}
\left\|x\left(h^{-}\right)\right\| & \leq\left\|(A+B K)^{h}\right\|\|x(0)\|+\|\Phi\|\|e(0)\| \\
& \leq \varepsilon_{K}\|x(0)\|+\|\Phi\|\|e(0)\| .
\end{aligned}
$$

Further,

$$
\begin{aligned}
\left\|x\left(2 h^{-}\right)\right\| & \leq \varepsilon_{K}\|x(h)\|+\|\Phi\|\|e(h)\| \\
& \leq \varepsilon_{K}\left(\varepsilon_{K}\|x(0)\|+\|\Phi\|\|e(0)\|\right)+\varepsilon_{L}\|\Phi\|\|e(0)\| \\
& =\varepsilon_{K}^{2}\|x(0)\|+\left(\varepsilon_{K}+\varepsilon_{L}\right)\|\Phi\|\|e(0)\|
\end{aligned}
$$

Since $\varepsilon_{K}+\varepsilon_{L}<1$ (also, $\varepsilon_{K}<1$ ) then we can see that

$$
\left\|x\left(2 h^{-}\right)\right\|<\varepsilon_{K}\|x(0)\|+\|\Phi\|\|e(0)\|
$$

By similar analysis, we obtain

$$
\left\|x\left(k_{\mu}^{-}\right)\right\|<\varepsilon_{K}\|x(0)\|+\|\Phi\|\|e(0)\|
$$

for $\mu=1,2, \ldots$. Finally, note that by the choice of initial conditions for the model and the observer we have that $\|e(0)\|<\gamma+W$ and the bound (36) follows.

The term $\|\Phi\|$ is a function of the update period $h$ and, in general, it increases as $h$ increases. Therefore, although the model-based networked system is asymptotically stable for any finite $h$, the performance of the system between update intervals deteriorates if we select a very long update interval. Hence, very large values of $h$ need to be avoided to obtain a desired inter-sample performance. 


\section{B. Piece-wise constant disturbances.}

We consider piece-wise constant disturbances $\|w(k)\| \leq W$ that satisfy

$$
w(k)=w\left(k_{d}\right), \quad k \in\left[k_{d}, k_{d+1}\right)
$$

where $h_{d}=k_{d+1}-k_{d}>p h$, for some integer $p>1$. In order to allow the observer to converge within some specified bounds we assume a synchronization of updates after disturbance changes. This simply means that if a change on the disturbance occurs at time $k_{d}$ then the next update time instant is given by $k_{\mu}=k_{d}+h$.

At the update times, the response of the system and the observer can be expressed as

$$
\left[\begin{array}{l}
x\left(k_{\mu}\right) \\
e\left(k_{\mu}\right)
\end{array}\right]=\Theta\left[\begin{array}{l}
x\left(k_{\mu-1}\right) \\
e\left(k_{\mu-1}\right)
\end{array}\right]
$$

for $k_{d}<k_{\mu}<k_{d+1}$, where

$$
\Theta=\left[\begin{array}{cc}
(A+B K)^{h} & \Phi \\
0 & \left(A_{e}-L C_{e}\right)^{h}
\end{array}\right] .
$$

Since all eigenvalues of matrix $\Theta$ have magnitude less than one, then there exists an integer $p\left(\varepsilon_{\Theta}\right)>0$ such that $\left\|\Theta^{p}\right\| \leq \varepsilon_{\Theta}$ for any $p \geq p\left(\varepsilon_{\Theta}\right)$.

Theorem 4. Assume that $\|x(0)\| \leq \gamma, \hat{x}(0)=0, \hat{w}(0)=w_{0}$, the observer is initialized as $\bar{x}(0)=0, \bar{w}(0)=w_{0}$ where $\left\|w_{0}\right\| \leq W$. Then, for piece-wise constant disturbances (41) the norm of the state is bounded by

$$
\|x(k)\| \leq \varepsilon_{K} \gamma+\|\Phi\|(\gamma+2 W)
$$

if $(A, B)$ is stabilizable, $(A, C)$ is observable, the nullspace of (6) contains only the zero vector, and $\varepsilon_{\Theta}$ and $\varepsilon_{L}$ satisfy

$$
\begin{gathered}
\varepsilon_{\Theta}<\frac{\gamma}{2\|\Gamma\|(\gamma+W)} \\
\varepsilon_{L}<\left(\frac{\gamma}{\gamma+2 W}\right)^{1 / p}
\end{gathered}
$$

Proof. Consider the response of the state at the time instant when the first change on the disturbance occurs which is represented by $h_{d}$

$$
\left\|x\left(h_{d}\right)\right\|=\left\|\Gamma(g)\left[\begin{array}{l}
x(p h) \\
e(p h)
\end{array}\right]\right\|=\left\|\Gamma(g) \Theta^{p}\left[\begin{array}{l}
x(0) \\
e(0)
\end{array}\right]\right\|
$$

where

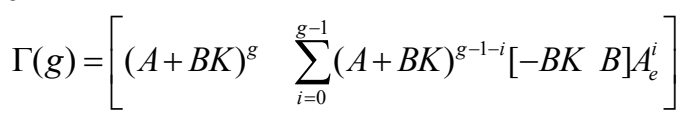

and $g=h_{d}-p h$. The initial conditions for the estimates of the disturbance are more general than in Corollary 3. This choice helps to deal with the worst possible variations on the disturbance, that is $\left\|w\left(k_{d}\right)-\bar{w}\left(k_{d}\right)\right\| \leq 2 W$ for any $k_{d}$, including $k_{d}=0$, which represents the first change since $w(0)$ is unknown. Then, we have that $\|e(0)\| \leq \gamma+2 W$ and (47) satisfies

$$
\left\|x\left(h_{d}\right)\right\| \leq 2\|\Gamma\| \varepsilon_{\Theta}(\gamma+W) .
$$

We want to find conditions on $\varepsilon_{\Theta}$ so $\left\|x\left(h_{d}\right)\right\|<\gamma$. Solving for $\varepsilon_{\Theta}$ in

$$
2\|\Gamma\| \varepsilon_{\Theta}(\gamma+W)<\gamma
$$

we obtain (45). Now, we want to guarantee that $\left\|e\left(h_{d}\right)\right\|<\gamma+2 W$. The error at time $k_{d}$ changes abruptly since a change on the disturbance occurred at that particular time instant. The error at time $h_{d}$ can be described by

$$
e\left(h_{d}\right)=e\left(h_{d}^{-}\right)-\left[\begin{array}{l}
0 \\
\tilde{w}\left(h_{d}\right)
\end{array}\right]
$$

where $\tilde{w}\left(h_{d}\right)=w\left(h_{d}\right)-w\left(h_{d}^{-}\right)$represents the variation of the disturbance at time $h_{d}$. Then, we have that

$$
\begin{aligned}
\left\|e\left(h_{d}\right)\right\| & \leq\left\|e\left(h_{d}^{-}\right)\right\|+2 W \leq \varepsilon_{L}^{p}\|e(0)\|+2 W \\
& \leq \varepsilon_{L}^{p}(\gamma+2 W)+2 W
\end{aligned}
$$

and we solve for $\varepsilon_{L}$ in

$$
\varepsilon_{L}^{p}(\gamma+2 W)+2 W<\gamma+2 W
$$

which results in condition (46). We conclude that at time $h_{d}$ we have both, $\left\|x\left(h_{d}\right)\right\|<\gamma$ and $\left\|e\left(h_{d}\right)\right\|<\gamma+2 W$, which are similar conditions to the initial state and initial observer error. The same analysis can be carried over (assuming synchronization of updates after every disturbance variation) to show that $\left\|x\left(k_{d}\right)\right\|<\gamma$ and $\left\|e\left(k_{d}\right)\right\|<\gamma+2 W$ for any $k_{d}$ such that $h_{d}=k_{d+1}-k_{d}>p h$.

Finally, following the results in Corollary 3 we can obtain the new performance bound for inter-sample intervals which considers the new choice of initial conditions and it is given by (44).

Remark. The results in this section consider stabilization (for nonzero initial conditions) and disturbance rejection (for constant or piece-wise constant disturbances). We also consider absence of feedback measurements for possibly long intervals of time and, since the initial conditions for the state observer and the model variables are uncertain, we obtain the performance bound (36) for constant disturbances which is determined based on the choice of initial states for the observer and the models which are selected as the zero states. Since no information is available about the initial states and the value of the uncertainty the previous choice is typical. For piece-wise constant disturbances we consider the effect of the worst possible change on the value of the disturbance which is $2 \mathrm{~W}$ and the performance bound (44) was obtained.

\section{EXAMPLE}

Consider the following open-loop unstable continuous-time system that represents the model of a batch reactor [20].

$$
A_{c}=\left[\begin{array}{cccc}
1.380 & -0.208 & 6.715 & -5.676 \\
-0.581 & -4.290 & 0 & 0.675 \\
1.067 & 4.273 & -6.654 & 5.893 \\
0.048 & 4.273 & 1.343 & -2.104
\end{array}\right]
$$



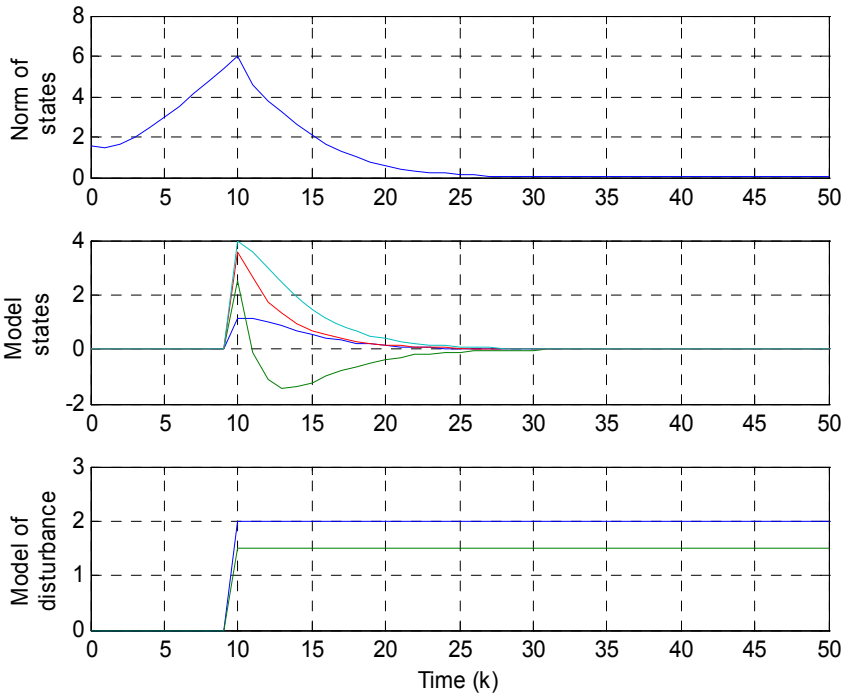

Fig. 2. $\|x(k)\|, \hat{x}(k), \hat{w}(k)$ for constant disturbance

$$
B_{c}=\left[\begin{array}{cc}
0 & 0 \\
5.679 & 0 \\
1.136 & -3.146 \\
1.136 & 0
\end{array}\right], \quad C=\left[\begin{array}{cccc}
1 & 0 & 1 & -1 \\
0 & 1 & 0 & 0
\end{array}\right]
$$

The continuous-time nominal parameters are discretized using $T=0.05$ seconds. The controller and observer gains for the discretized system are given by:

$$
\begin{aligned}
K & =\left[\begin{array}{cccc}
2.366 & -1.693 & 1.443 & -3.059 \\
12.835 & -1.485 & 9.296 & 9.623
\end{array}\right], \\
L & =\left[\begin{array}{cccccc}
-14.962 & 0.019 & -21.921 & -39.467 & 3.259 & -43.453 \\
-61.524 & 2.468 & -277.223 & -339.266 & 41.754 & -189.879
\end{array}\right]^{T}
\end{aligned}
$$

In order to reduce communication and to obtain good transient performance between updates we select $h=10$. We obtain that $\left\|(A+B K)^{h}\right\|=0.0028$ and $\left\|\left(A_{e}-L C_{e}\right)^{h}\right\|=0.1762$ so we can readily check that $\varepsilon_{K}+\varepsilon_{L}<1$. For the selected update period we have $\|\Phi\|=3.9321$. The initial conditions are given by $x(0)=\left[\begin{array}{llll}1.1 & -0.5 & 0.4 & 0.9\end{array}\right]^{T}$. Using $\gamma=\|x(0)\|$ we have that he right hand side of (36) is approximately 17.25, for $W=2.83$ (both components of the disturbance are allowed to take values between -2 and 2).

Fig. 2 shows the norm of the state of the system for constant disturbance $w=\left[\begin{array}{ll}2 & 1.5\end{array}\right]^{T}$. The maximum peak of $\|x(k)\|<17.25$ is reached at $k=10$, at this time instant the first set of observed states and disturbance are received at the controller node which significantly improve the response of the system from that point forward. Fig. 2 also shows the model variables, the estimates of the model states $\hat{x}(k)$ and the estimates of the disturbance $\hat{w}(k)$ as seen by the controller node.

\section{CONCLUSION}

A model-based approach for networked systems perturbed by external disturbances was discussed in this paper. An augmented state observer was used in order to estimate both plant states and the external disturbance. Further, analysis of system performance between update time instants was addressed. This analysis provided insight into the appropriate selection of update time intervals in order to obtain a satisfactory inter-update performance.

\section{REFERENCES}

[1] P. J. Antsaklis, J. Baillieul, "Special issue on technology of networked control systems," Proc. IEEE, vol. 95, no.1, 2007.

[2] M. B. G. Cloosterman, L. Hetel, N. van de Wouw, W. P. M. H.Heemels, J. Daafouz, H. Nijmeijer, "Controller synthesis for networked systems," Automatica, vol. 46, pp. 1584-1594, 2010.

[3] A. H. Tahoun, H. Fang, "Adaptive stabilization of networked control systems with delays and state-dependent disturbances," in Proceedings of 17th Mediterranean Conference on Control and Automation, 2009, pp. 622-627.

[4] A. Cervin, T. Henningson, "Scheduling of event-triggered controllers on a shared network," in Proceedings of the 47th IEEE Conference on Decision and Control, 2008, pp. 3601-3606.

[5] L. A. Montestruque, P. J. Antsaklis, "On the model-based control of networked systems," Automatica, vol. 39, no. 10, pp. 1837-1843, 2003.

[6] L. A. Montestruque, P. J. Antsaklis, "Stability of model-based control of networked systems with time varying transmission times," IEEE Transactions on Automatic Control, vol. 49, no. 9, pp. 1562-1572, 2004.

[7] E. Garcia and P. J. Antsaklis, "Model-based event-triggered control for systems with quantization and time-varying network delays," IEEE Transactions on Automatic Control, vol. 58, no.2, pp. 422-434, 2013.

[8] E. Garcia and P. J. Antsaklis, "Model-based control of networked distributed systems with multi-rate state feedback updates," International Journal of Control, vol. 86, no.9, pp. 1503-1517, 2013.

[9] E. Garcia, P. J. Antsaklis, "Adaptive stabilization of Model-Based Networked Control Systems," in Proceedings of the American Control Conference, 2011, pp. 1094-1099.

[10] E. Garcia and P. J. Antsaklis "Decentralized model-based eventtriggered control of networked systems," in Proceedings of the American Control Conference, 2012, pp. 6485-6490.

[11] G. Conte, A. M. Perdon, G. Vitaioli, "A model based control scheme with sampled information," in Proceedings of the 17th Mediterranean Conference on Control and Automation, 2009, pp. 1354-1360.

[12] J. Lunze, D. Lehmann, "A state-feedback approach to event-based control," Automatica, vol. 46, no. 1, pp. 211-215, 2010.

[13] D. Lehmann, J. Lunze, "Event-based output feedback control," in Proceedings of the 19th Mediterranean Conference on Control and Automation, 2011, pp. 982-987.

[14] M. Yu, L. Wang, T. Chu, G. Xie, "Stabilization of networked control systems with data packet dropout and network delays via switching system approach," in Proceedings of the 43rd IEEE Conference on Decision and Control, 2004, pp. 3539-3544.

[15] H. Yu, A. Wang, Y. Zheng, "On the model-based networked control for singularly perturbed systems," Control Theory and Applications, vol. 6, no. 2, pp. 153-162, 2008.

[16] Y. Sun, N. H. El-Farra, "Quasi-decentralized model-based networked control of process systems," Computers and Chemical Engineering, vol. 32, pp. 2016-2029, 2008.

[17] M. Iwase, S. Hatakeyama and K. Furuta. "Analysis of Intermittent Control Systems by Human Operations," IECON 2006, 32nd Annual Conference on IEEE Industrial Electronics, 2006, pp. 4516-1521.

[18] E. Garcia and P. J. Antsaklis, "Output feedback networked control with persistent disturbance attenuation," Systems and Control Letters, vol. 62, pp. 943-948, 2013.

[19] P. J. Antsaklis, A. Michel, Linear systems, first ed., McGraw Hill, New York, 1997.

[20] M.C.F. Donkers, W.P.M.H Heemels, N. Wouw, L. Hetel, "Stability analysis of networked control systems using a switched linear systems approach," IEEE Transactions on Automatic Control, vol. 56, pp. 2101-2115, 2011. 